\title{
OS EFEITOS DE UM PROGRAMA DE ATIVIDADE FÍSICA NA PRESSÃO ARTERIAL E FREQUÊNCIA CARDÍACA DE PACIENTES HIPERTENSOS E DEFICIENTES FÍSICOS
}

\author{
THE EFFECTS OF A PROGRAM OF PHYSICAL ACTIVITY ON THE BLOOD AND HEART \\ PRESSURE OF HYPERTENSIVE PATIENTS AND PHYSICAL DEFICIENTS \\ Laila Cristina Moreira Damázio ${ }^{\mathbf{a}^{*}}$, Gustavo Ribeiro de Souza Filho ${ }^{\mathbf{b}^{*}}$, \\ Amanda Chinellato de Lima Pereira ${ }^{{ }^{*}}$, Thaís Lany de Oliveira Leão ${ }^{\mathrm{d}^{*}}$, Lívia Candian Ferreira ${ }^{\mathrm{e}^{*}}$, \\ Felipe Nunes Mourão ${ }^{\mathrm{f}^{*}}$ \\ lailacmdamazio@gmail.comª, gusouzaf@hotmail.com ${ }^{\mathrm{b}}$, amandapereira612@hotmail.comc, thaisloleao@gmail.com ${ }^{\mathrm{d}}$, livinhacandian@hotmail.com, \\ felipenunesmourao@gmail.com ${ }^{\mathrm{f}}$ \\ UFSJ/MEDICINA/CDB*
}

Data de recebimento do artigo: 12/01/2019 Data de aceite do artigo: 18/02/2019

\section{RESUMO}

Objetivo: Investigar os efeitos de um programa de atividade física, com intensidade moderada, na pressão arterial e frequência cardíaca dos pacientes com deficiência física e hipertensão arterial em um município do interior de Minas Gerais/Brasil. Métodos: Foram utilizados 21 pacientes, sendo 11 do gênero feminino e 10 do gênero masculino. Desses, 6 são hipertensos e 9 deficientes físicos. Os dados foram coletados todos os dias antes do paciente realizar o exercício físico e após a realização do mesmo, sendo aferida sua pressão arterial e frequência cardíaca nos dois momentos. O programa de atividade física, ocorreu em uma academia adaptada, onde os pacientes exercitavam 3 vezes por dia, durante 50 minutos, por 6 meses. Em seguida, os valores foram analisados com auxílio do programa GraphPad Prism 5.0, utilizando o teste estatístico $t$-student e ANOVA. Resultados: Os dados demonstraram que ocorreu redução da pressão sistólica e frequência cardíaca de todos os pacientes após a prática de exercícios físicos. Quando analisados isoladamente, os grupos de idosos sem comorbidades e deficientes apresentaram uma melhora na pressão sistólica antes do exercício, enquanto o grupo de hipertensos apresentou melhora da pressão sistólica após a atividade física. Já a frequência cardíaca dos hipertensos apresentou uma queda após o exercício físico. Conclusão: O presente estudo demonstra que um programa de atividade física moderado foi eficaz para a redução dos níveis pressóricos e da frequência cardíaca de indivíduos hipertensos e com deficiência.

Palavras-chave: Hipertensão, Pessoas com Deficiência, Pressão Arterial, Frequência Cardíaca, Exercício.

\section{ABSTRACT}

Objective: To investigate the effects of a physical activity program with moderate intensity on blood pressure and heart rate of patients with physical disability and arterial hypertension in a municipality in the interior of Minas Gerais / Brazil. Methods: Twenty-one patients were used, of which 11 were female and 10 were male. Of these, 6 are hypertensive and 9 are physically disabled. The data were collected every day before the patient performed the physical exercise and after the exercise, and their blood pressure and heart rate were measured at both moments. The physical activity program took place in an adapted gym, where patients exercised 3 times a day for 50 minutes for 6 months. Then, the values were analyzed with the aid of the GraphPad Prism 5.0 program, using the statistical test t-student and ANOVA. Results: The data demonstrated that there was reduction of systolic pressure and heart rate of all patients after physical exercise. When analyzed in isolation, the groups of elderly patients with no comorbidities and deficits showed an improvement in systolic pressure before exercise, while the group of hypertensive patients presented improvement in systolic pressure after physical activity. On the other hand, the heart rate of hypertensive patients showed a decrease after exercise. Conclusion: The present study demonstrates that a moderate physical activity program was effective in reducing pressure levels and heart rate in hypertensive and disabled individuals.

Keywords: Hypertension, People With Disabilities, Blood Pressure, Heart Rate, Exercise. 


\section{Introdução}

O envelhecimento populacional é hoje um dos maiores desafios da saúde pública. Este fenômeno teve início em países desenvolvidos, entretanto, mais recentemente, é nos países em desenvolvimento que essa etapa da transição demográfica tem ocorrido de forma mais acentuada. No Brasil, por exemplo, o número de idosos, considerando-se como idoso todo indivíduo com idade maior ou igual a 60 anos, passou de 3 milhões em 1960, para 7 milhões em 1975 e 14 milhões em 2002, indicando um aumento de 500\% em quarenta anos. Estima-se que esse dado alcançará o valor de 32 milhões em 2020. ${ }^{1}$

Paralelamente às alterações evidentes na pirâmide populacional, patologias próprias de idades mais avançadas ganham uma expressão significativa no conjunto da sociedade. As doenças crônicas não-transmissíveis (DCNT) têm uma prevalência aumentada na população, proporcional ao aumento da idade. As DCNT comprometem a qualidade de vida das pessoas idosas, tornando-as mais dependentes e mais propensas a outras doenças. A consequência evidente desse contexto é um aumento na demanda por serviços de saúde ${ }^{2}$.

As principais doenças crônicas que afetam a população acima dos 60 anos no Brasil são a hipertensão e o diabetes. A prevalência estimada de hipertensão arterial sistêmica (HAS), na população adulta, no Brasil é em torno de 35\%. Esse valor é proporcional à idade, chegando a cerca de $75 \%$ na população acima de 70 anos ${ }^{3}$. Já com relação a diabetes, a proporção é de $14,5 \%$ entre 60 a 64 anos, $19,9 \%$ entre 65 a 74 anos e 19,6\% para pacientes acima de 75 anos ${ }^{4}$.

Nesse âmbito é evidente que essas doenças crônicas prevalentes em idosos se transformaram em um grande problema de saúde pública, talvez o maior da atualidade. Dessa forma, a atividade física se mostra de extrema relevância para tratar o problema dessa população em específico. A atividade física regular nos idosos, particularmente os exercícios nos quais se sustenta o próprio peso e exercícios de força, promovem maior fixação de cálcio nos ossos, auxiliando na prevenção e no tratamento da osteoporose. A prática de exercício físico aumenta a força e a endurance musculares, o equilíbrio e a flexibilidade, com a consequente di- minuição da incidência de quedas, fraturas e suas complicações. Os idosos portadores de osteoartrose também podem e devem praticar atividade física regular, desde que adaptada à sua condição ${ }^{5}$.

Estar ativo fisicamente é um elemento primordial para a longevidade. A prática de atividade física auxilia as pessoas a manterem seus corpos em forma e a reduzirem o peso corporal diminuindo o risco de doenças coronarianas. Isso resulta na manutenção de uma pressão sanguínea moderadamente baixa e redução do colesterol total e das lipoproteínas de baixa densidade (LDL), assim como o aumento das lipoproteínas de alta densidade (HDL). Essas mudanças, em conjunto, contribuem para reduzir o número de ataques cardíacos, acidentes vasculares cerebrais e doenças renais. Esses benefícios apresentados são capazes de proporcionar melhora da qualidade de vida para jovens, adultos e proporciona contribuições importantes para as pessoas da terceira idade ${ }^{6}$.

Os aspectos positivos dos exercícios físicos e o bom condicionamento físico total também se aplicam a redução dos riscos para alguns distúrbios metabólicos crônicos associados à obesidade, tais como resistência à insulina e diabetes tipo II. A prática de exercícios moderados, mesmo na ausência de redução de peso significativa, tem demonstrado melhora na sensibilidade à insulina e redução da necessidade de tratamento com insulina em pacientes com diabetes tipo II ${ }^{6}$.

Dessa forma, torna-se necessário o estudo sobre os efeitos da prática de atividade física moderada na pressão arterial e frequência cardíaca de idosos com hipertensão arterial e deficiências, no município de São João del-Rei/Minas Gerais.

\section{Métodos}

\section{TIPO DE ESTUDO E LOCAL}

O presente estudo trata de um estudo analítico, do tipo longitudinal e prospectivo, realizado no Laboratório de Pesquisa e Intervenção Psicossocial (LAPIP) do Departamento de Psicologia da Universidade Federal de São João Del Rei. O LAPIP foi criado em junho de 2010 e é utilizado como laboratório para a Unidade Curricular de Educação Física Adaptada. Esse laboratório tem se desenvolvido de forma relevante na área de pes- 
quisa e extensão e oferta um amplo atendimento a usuários externos à universidade por meio de projetos com grupos, instituições e comunidades em um município do interior de Minas Gerais/Brasil.

\section{AMOSTRAGEM}

$\mathrm{Na}$ pesquisa foram incluídos 21 pacientes, sendo 11 do gênero feminino e 10 do gênero masculino, sendo que, desses, 6 são hipertensos e 9 deficientes físicos. Foram incluídos na amostra todos os pacientes que assinaram o Termo de Consentimento Livre e Esclarecido (Resolução 466/2012 e Norma Operacional 001/2013) e que participaram do programa de práticas de atividade física no LAPIP, seguindo orientações dos profissionais e estudantes de educação física. Foram excluídos da amostra os pacientes que não atingiram uma frequência mínima durante as práticas, e ainda pacientes caracterizados como portadores de Hipertensão Arterial Sistêmica Grave.

\section{INSTRUMENTOS E PROCEDIMENTOS}

Todos os dias, antes de iniciarem o programa de atividades físicas no LAPIP, cada paciente teve sua pressão arterial aferida de acordo com a técnica indicada pela Sociedade Brasileira de Cardiologia (2010). Foi solicitado a cada paciente que se mantivesse sentado, com os pés apoiados sobre o chão, o braço esquerdo relaxado, apoiado sobre uma mesa na altura do coração e com a palma da mão voltada para cima. Para a realização do exame foram utilizados um esfigmomanômetro aneroide, devidamente calibrado, e um estetoscópio, ambos da marca Premium, além de um relógio.

A frequência cardíaca do paciente também foi avaliada, de acordo com a técnica indicada por Porto $^{7}$. O examinador utilizou a mão direita para palpar o pulso esquerdo do paciente. Para isso, empregam-se as polpas dos dedos indicador e médio próximos à cabeça do osso rádio, variando a força de compressão até que seja obtido o pulso máximo. O polegar se posiciona delicadamente no dorso do punho do paciente como um apoio. A mão do paciente deverá estar em posição supina, posicionada sobre a mesa. O examinador conta, então, quantos pulsos sentiu, durante 15 segundos. O resultado é multiplicado por 4, para transformar a o valor obtido em frequência cardíaca, ou seja, número de pulsos por minuto.
A pressão arterial e a frequência cardíaca foram reavaliadas 5 minutos após o término dos exercícios de cada sessão, para que os dados não sofressem interferência da atividade física realizada.

O programa de exercícios físicos foi realizado por 6 meses, sendo realizado 3 vezes por semana, durante 50 minutos de atividade por dia. $\mathrm{O}$ programa incluiu exercícios dinâmicos e ativos com os membros superiores e inferiores, utilizando bastões, bolas e aparelhos de musculação. Os exercícios são, basicamente, de fortalecimento e alongamento dos músculos de todo o corpo, além de exercícios aeróbicos. O programa de atividade física moderado, considerando VO2máx. entre 60 a 70\%. Esses exercícios foram realizados nos equipamentos de musculação do LAPIP, exercícios na cadeira extensora, flexora, leg press, desenvolvimento, cicloergômetro, esteira e bicicleta ergométrica.

O programa de atividade física foi monitorado por acadêmicos dos cursos de medicina e educação física, além de professores com formação em educação física, fisioterapia e medicina. O projeto de pesquisa foi submetido ao comitê de ética em pesquisa com seres humanos da UFSJ, apresentado o CAAE de aprovação número 54985416900005151.

Para análise dos dados foi utilizado o programa estatístico GraphPad Prism 5.0, utilizando como teste estatístico o Teste $t$-student não pareado e ANOVA, considerando como nível de significância $\mathrm{p}<0,05$.

\section{Resultados}

Os resultados demonstraram que não ocorreu alteração significativa na pressão arterial diastólica (PAD) antes $(\mathrm{p}=0,5821 \pm 2,232)$ ou após $(\mathrm{p}=0,611 \pm 1,76)$ os pacientes realizarem o exercício físico, ao comparar o início com o final do projeto. Antes da atividade física, também não foi evidenciado diferença significativa na pressão arterial sistólica (PAS) ( $\mathrm{p}=0,5135 \pm 3,579)$ dos pacientes como um todo. Porém, foi evidenciada redução significativa na PAS $(\mathrm{p}=0,0005 \pm 2,39)$ após realizarem atividade física, quando comparados os valores no início do projeto com o final.

Os resultados da frequência cardíaca demonstraram que ocorreu diminuição sig- 
nificativa após realizarem a atividade física $(\mathrm{p}=0,0013 \pm 2,09)$, quando comparados no início com o final do projeto.

Ao analisar a média da variação da frequência cardíaca entre os grupos, antes dos exercícios físicos e ao longo do projeto, demonstrou-se uma diferença significativa ( $p=0,0167 ; F=5,1)$. Os resultados da frequência cardíaca depois da atividade física também demonstraram diferença significativa $(\mathrm{p}=0,0378 ; \mathrm{F}=3,875$.

Os resultados demonstraram que ocorreu alteração significativa na PAD $(\mathrm{p}=0,0009 ; \mathrm{F}=11,22)$, entre os grupos durante o projeto, antes de se realizar o exercício (Figura 1).

Figura 1. Média da pressão arterial diastólica (PAD) entre os grupos experimentais ao longo do programa, antes do exercício físico.

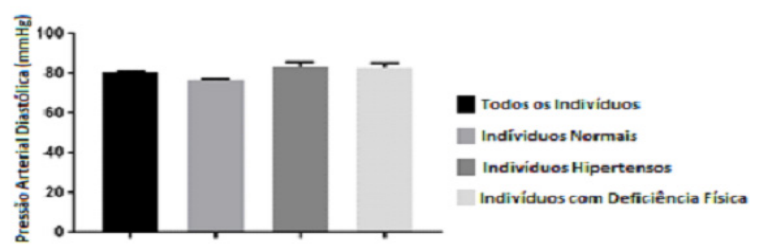

Fonte: autores

Os resultados demonstraram que ocorreu alteração significativa na PAS $(\mathrm{p}=0,0436 ; \mathrm{F}=3,675)$ entre os grupos antes da atividade física, ao longo do projeto. Sendo que, a PAS depois do programa de atividade física não sofreu alteração $(\mathrm{p}=0,1609$; $\mathrm{F}=2,408$ ) entre os grupos.

Os resultados demonstraram que não ocorreu alteração significativa na frequência cardíaca dos pacientes sem comorbidades clínicas, antes $(\mathrm{p}=0,3965 \pm 3,969)$ e depois $(\mathrm{p}=0,1038 \pm 1,937)$ dos exercícios físicos, ao comparar o início com o final do projeto.

A análise da frequência cardíaca dos indivíduos hipertensos não demonstrou alteração significativa antes $(\mathrm{p}=0,5257 \pm 4,89)$ da atividade física, em comparação com o início do projeto e o final. No entanto, após o exercício físico foi evidenciado alteração significativa ( $\mathrm{p}=0,0409 \pm 5,842$; Figura 2) quando comparados os indivíduos hipertensos antes do projeto com os mesmos indivíduos após a execução do projeto.
Figura 2. Média da frequência cardíaca dos indivíduos hipertensos depois da atividade física

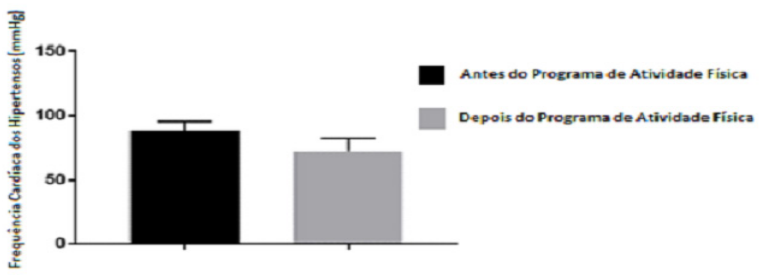

Fonte: autores

Os resultados demonstraram que não ocorreram alterações significativas na frequência cardíaca dos indivíduos com deficiência física antes $(\mathrm{p}=0,4428 \pm 5,602)$ e após $(\mathrm{p}>0,9999 \pm 5,842)$ realizarem os exercícios físicos.

Os resultados demonstraram que não ocorreram alterações significativas na $\mathrm{PAD}$, antes da prática dos exercícios físicos, entre os indivíduos sem comorbidades clínicas $(\mathrm{p}=0,8864 \pm 3,768)$ e entre os hipertensos $(\mathrm{p}=0,0631 \pm 4,549)$ ao se comparar o início do projeto com o final. Não ocorrendo também alteração significativa na PAD ( $\mathrm{p}=0,6109 \pm 3,073)$ depois do exercício físico nos pacientes hipertensos. O mesmo ocorreu com os resultados na PAD $(\mathrm{p}=0,4341 \pm 4,902)$ antes do exercício físico, nos pacientes deficientes. Entre os indivíduos com deficiência física não foi encontrado alteração significativa na PAD $(\mathrm{p}=0,1801 \pm 3,745)$ antes do exercício físico quando comparados no início do projeto com o final.

Quanto aos dados da PAS foi evidenciado entre os pacientes sem comorbidades clínicas que não ocorreu alteração significativa na PAS $(\mathrm{p}=0,3122 \pm 4,12)$ antes do exercício físico, ao compará-los no início do projeto e ao final do projeto. Porém, o mesmo grupo apresentou alteração significativa na PAS $(\mathrm{p}=0,0207 \pm 3,093)$ depois do exercício físico (Figura 3 ).

Figura 3. Média da pressão arterial sistólica no grupo de indivíduos sem comorbidades clínicas após a prática do exercício físico.

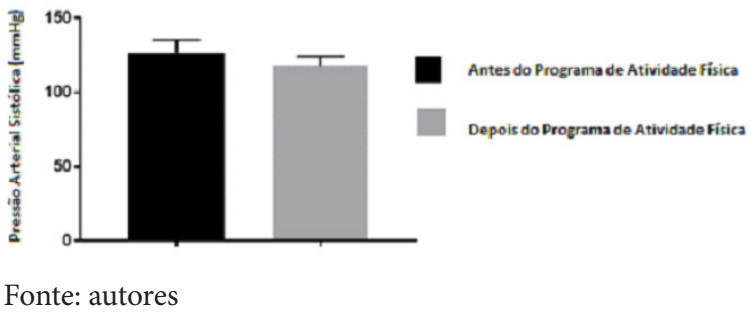


Os resultados demonstraram que não ocorreu alteração significativa na PAS $(p=0,6734 \pm 11,18)$ entre os indivíduos hipertensos antes do exercício físico ao comparar o início com o final do projeto. Todavia, ao analisar a variação da PAS após o exercício físico nos pacientes hipertensos, constatou-se que houve uma alteração significativa $(\mathrm{p}=0,0071 \pm 3,416)$ quando comparados no início com o final do projeto (Figura 4).

Figura 4. Média da pressão arterial sistólica no grupo de indivíduos com hipertensão após a prática dos exercícios físicos

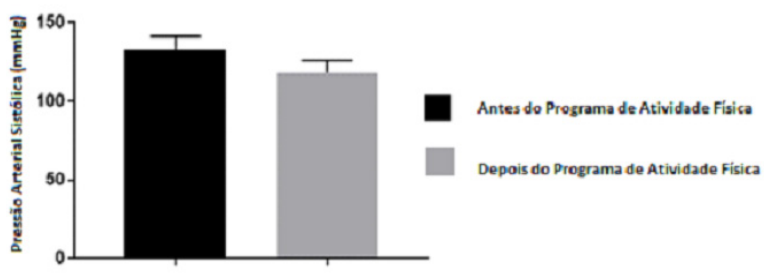

Fonte: autores

Os resultados demonstraram que ocorreu alteração significativa na PAS $(\mathrm{p}=0,0335 \pm 4,014)$ entre os indivíduos com deficiência física, antes do exercício físico (Figura 5). Contudo não ocorreu alteração significativa na PAS ( $\mathrm{p}=0,1099 \pm 6,009)$ depois do exercício físico no mesmo grupo comparando-se o início com o final do projeto.

Figura 5. Média da pressão arterial sistólica entre os indivíduos do grupo de deficientes físicos antes da prática do exercício

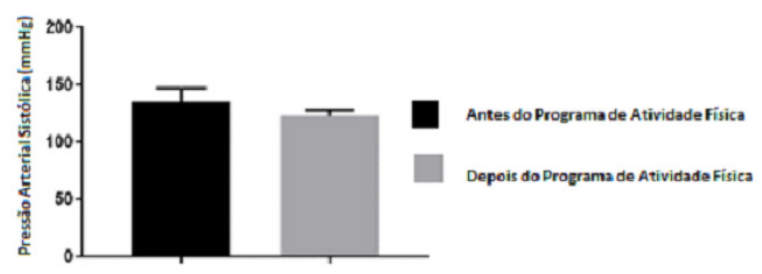

Fonte: autores

\section{Discussão}

A partir da metodologia empregada no estudo encontrou-se um efeito homogêneo da intervenção do exercício físico sobre os valores de pressão arterial sistólica (PAS) e frequência cardíaca (FC). A população estudada apresentou redução dos dois parâmetros depois da realização da ativida- de física e os grupos dos deficientes e das pessoas sem comorbidades apresentaram ainda redução da PAS antes do exercício.

É inquestionável o papel da terapia medicamentosa em pacientes com HAS (hipertensão arterial sistêmica). Os antihipertensivos são os principais responsáveis pela redução significativa e efetiva dos níveis pressóricos, e da mortalidade causada por esses níveis. Contudo, cada vez mais vem sendo estimulada uma abordagem não medicamentosa para pacientes hipertensos de grau leve a moderado, como opção para redução ou, até mesmo, normalização dos níveis de pressão arterial. Dentre as condutas não medicamentosas, a prática regular de exercícios físicos vem sendo recomendada por profissionais da saúde como uma maneira efetiva no controle dos níveis de pressão arterial em pacientes hipertensos de graus leve e moderada, inclusive daqueles pacientes que se encontram em vigência da terapêutica farmacológica ${ }^{8}$.

A prática de atividade física exerce um efeito agudo e crônico sobre a pressão arterial, isso devido aos efeitos hipotensores da prática do exercício. Dentre os efeitos agudos podemos apontar a redução imediatamente após uma sessão de exercício físico aeróbico, denominada hipotensão pós-exercício. No presente estudo foi possível identificar uma redução da pressão arterial sistólica dos hipertensos depois do exercício, assim como dos normotensos e deficientes físicos. Entretanto, alguns fatores, como o tempo da sessão e o nível inicial da pressão arterial podem influenciar nessa resposta precoce. O exercício mais prolongado tende a provocar uma diminuição mais duradoura e maior do que um exercício de curta duração. Outro fator que influencia, de forma importante, é o nível de pressão arterial inicial. O efeito hipotensor do exercício tende a ser mais expressivo em pessoas que partem de valores iniciais de pressão arterial mais elevados, no caso dos pacientes hipertensos, entretanto, a prática foi igualmente benéfica para todos os indivíduos da pesquisa ${ }^{9}$.

Com relação à intensidade do exercício, quanto maior o consumo máximo de oxigênio ocorrido na sessão, maior será o efeito da queda na pressão arterial após o exercício. Apesar do exercício provocar uma redução na pressão arterial nos momentos subsequentes ao mesmo, um aspecto importante a ser 
considerado é por quanto tempo este efeito perdura. Só terá implicação clínica para o paciente se o efeito hipotensor do exercício perdurar por longo período ${ }^{8}$.

Dois mecanismos hemodinâmicos têm sido apontados como possíveis responsáveis pela hipotensão pós-exercício: 1) diminuição do débito cardíaco ou 2) diminuição da resistência vascular periférica. No presente estudo, nos pacientes hipertensos idosos, o mecanismo responsável pela diminuição da pressão arterial pós-exercício possivelmente está relacionado à diminuição do débito cardíaco devido a um menor volume sistólico. Além disso, observa-se que o menor volume sistólico é devido a um menor enchimento ventricular (volume diastólico final), desde que, tanto a função sistólica quanto a função diastólica não são alteradas, no período pós-exercício, em relação ao período pré-exercício ${ }^{8}$.

Com relação aos pacientes sem comorbidades, participantes do estudo, houve redução da pressão sistólica antes do exercício, representando o efeito hipotensor longitudinal provocado pela prática de atividade física. É importante ressaltar a efetividade dessa redução, mesmo que de maneira menos intensa, em pessoas com níveis pressóricos menores, dentro do padrão de normalidade.

Portanto, as alterações dos níveis pressóricos pelo exercício são resultados de adaptações anatômicas e fisiológicas, como a redução do débito cardíaco, da atividade simpática e da resistência vascular e resistência à insulina. Essas mudanças fisiológicas promovem a redução dos níveis pressóricos, verificados especialmente na pressão arterial sistólica, não sendo significativas as alterações de pressão arterial diastólica. No presente estudo, esta redução foi mais expressiva e significante após o terceiro mês e não foi associada com a diminuição na pressão arterial média (PAM) e pressão arterial diastólica (PAD), o que sugere que um período mais longo de exercício poderia alterar efetivamente a pressão arterial. ${ }^{10}$

A realização de exercícios físicos a longo prazo provoca a redução da atividade simpática sobre o coração, o que leva a uma redução da frequência cardíaca, como observada na população estudada. Essa diminuição do tônus simpático representa na realidade um efeito de normalização quando comparado a indivíduos normotensos. É importante ressaltar, entretanto, a diferença dos mecanismos de redução da FC em indivíduos normotensos e indivíduos hipertensos, uma vez que, nos primeiros ela ocorre por uma adaptação intrínseca ou de marca-passo e nos segundos ela é devida a uma adaptação de redução do tônus simpático, ou seja, a uma tentativa de adequar sua atividade aos padrões de normalidade ${ }^{11}$.

No presente estudo, os pacientes com deficiência física apresentavam sobrepeso significativo. Isso possibilitou uma análise no sentido de distúrbios alimentares em pacientes deficientes e sua relação direta com a limitação física, uma vez que, todos possuíam o cognitivo preservado. Mesmo com a restrição de movimentos e o sobrepeso verificado, a prática de exercícios planejada e guiada por orientadores físicos mostrou-se eficaz na redução longitudinal da pressão arterial observada nas medições anteriores à prática da atividade física.

\section{Conclusão}

Conclui-se que o programa de atividade física moderado, em uma academia de musculação adaptada beneficiou os pacientes com hipertensão arterial e deficiência física. No entanto, é necessário que o programa de exercício físico seja prolongado e orientado para que os benefícios sejam mantidos.

\section{Referências}

1. Contessa NRK. Envelhecimento e saúde da pessoa idosa - espaço e possibilidades no hospital universitário Polydoro Ernani de São Thiago. Universidade Federal de Santa Catarina: Florianópolis, 2010.

2. Lima-Costa MF, Veras R. Saúde pública e envelhecimento. Cad. Saúde Pública. 2003 mai-jun; 19(3):700-701.

3. Daut CVG. Fatores de risco de Doenças Crônicas Não Transmissíveis em uma comunidade universitária do Rio Grande do Sul do Brasil. Universidade Federal do Rio Grande do Sul (UFRGS). Porto Alegre; 2013.

4. Brasil. Ministério da Saúde. 57,4 milhões de brasileiros têm pelo menos uma doença crônica. Brasília: Ministério da Saúde; 2014.

5. Nobrega ACL, Freitas EV, Oliveira MAB, Leitão MB, Lazzoli JK, Nahas RM, et al. Posicionamento oficial da Sociedade Brasileira de Medicina do Esporte e da Sociedade Brasileira de Geriatria e Gerontologia: atividade física e saúde no idoso. Rev Bras Med Esporte. 1999; 5(6): 207-211. 
6. Guyton AC, Hall JE. Tratado de Fisiologia Médica. 11 ed. Rio de Janeiro: Elsevier; 2006.

7. Porto CC. Semiologia Médica. 7 ed. Guanabara Koogan; 2013.

8. Laterza MC, Rondon MUPB, Negrão CE. Efeitos do Exercício Físico Aeróbio na Hipertensão Arterial. Rev Soc Card Rio Grande do Sul. 2006 Set/Out/Nov/Dez; XV(9): 1-9. Goessler KF, Martins-Pinge MC, Cunha NV, Karlen-Amarante M, Polito MD. Hipertensão arterial e exercício físico aeróbio. Medicina (Ribeirão Preto) 2015;48(1):87-98.

9. Monteiro LH, Rolim LMC, Squinca DA, Silva FC, Ticianeli CCC, Amaral SL et al. Efetividade de um programa de exercícios no condicionamento físico, perfil metabólico e pressão arterial de pacientes hipertensos. Rev Bras Med Esporte. 2007 Mar/Abr; 13(2): 107-112.

10. Negrão CE, Rondon MUPB. Exercício físico, hipertensão e controle barorreflexo da pressão arterial. Rev Bras Hipertens. 2001 janeiro/março; 8(1): 89-96. pressão arterial e de cardíaca de pacientes hipertensos e deficientes físicos. Rev. Aten. Saúde. 2019;17(59):81-87. 